\title{
Modélisation des mises en suspension dans le détroit du Pas-de-Calais sous l'influence des courants de marée et de la houle.
}

\author{
Nicolas GUILLOU ${ }^{1}$ - Centre d'Etude Techniques Maritimes et Fluviales / \\ UMR CNRS - UBO 6539 LEMAR - Institut Universitaire Européen de la \\ Mer, 29280 Plouzané, France. Nicolas.Guillou@univ-brest.fr, \\ Nicolas.Guillou@equipement.gouv.fr \\ Georges CHAPALAIN² - UMR CNRS - UBO 6539 LEMAR - IUEM, \\ 29280 Plouzané, France. Georges.Chapalain@univ-brest.fr \\ ${ }^{1}$ Ingénieur chargé d'études, Doctorant en hydraulique maritime et dynamique \\ sédimentaire, ${ }^{2}$ Chercheur CNRS
}

\section{Résumé}

Un modèle hydro-sédimentaire tridimensionnel basé sur le code COHERENS [Luyten et al., 1999] a été appliqué à l'environnement du détroit du Pas-de-Calais et des zones côtières adjacentes. Le modèle reproduit les mesures de courant, de turbulence et de turbidité dans une zone de faible profondeur située au large des plages de Hardelot et de Merlimont. La répartition spatiale des mises en suspension est étudiée sur un cycle de marée moyenne. L'influence d'une houle de tempête est analysée sur la base d'un modèle de propagation spectrale et d'un modèle d'interaction houle-courant en couche limite benthique.

\begin{abstract}
A three-dimensionnal model based on COHERENS [Luyten et al., 1999] is used to simulate the suspended sediment transport in the Dover Straits and its coastal adjacent areas. The model reproduces measurements of current, turbulence and concentration at two shallow water sites off Hardelot and Merlimont beaches. The pattern of sediment resuspension is presented on a mean tide cycle. The influence of surface gravity waves is investigated with the use of a spectral wave propagation model and a model of wave-current interaction in the bottom boundary layer.
\end{abstract}

Mots clés : modélisation, mesures, transport sédimentaire multiclasse, interaction houle-courant en couche limite benthique.

\section{Introduction}

Le détroit du Pas-de-Calais est un passage étroit d'une largeur minimale de $32 \mathrm{~km}$ qui met en communication la partie orientale de la Manche et le sud de la mer du Nord. Les faibles profondeurs ( $40 \mathrm{~m}$ en moyenne) favorisent de fortes interactions non-linéaires entre les ondes de marée [Le Provost et Fornerino, 1985] générant un hydrodynamisme fort. En marée de vive-eau, les courants atteignent ainsi 4 nœuds aux abords du cap Gris-Nez. Les tempêtes, dans cette région, sont également susceptibles d'intensifier cet hydrodynamisme. De telles conditions font du détroit du Pas-de-Calais une zone marquée par une érosion intense du lit 
et des transports sédimentaires conséquents. Si d'autres sources (rejets de particules par les cours d'eau, érosion des côtes...) viennent alimenter les flux sédimentaires dans le détroit, les mises en suspension de la couverture superficielle en constituent l'apport majeur [Eisma et Kalf, 1979]. Ces mouvements sont notamment révélés lorsque les fortes mises en suspension atteignent la surface. La figure 1 est une image satellitale de réflectance de surface de la Manche Orientale prise le 17 Mars 1990 à 12 :22 GMT [Kergomard et al., 1993]. La côte anglaise est caractérisée par de fortes turbidités liées aux mises en suspension des sédiments fins présents au sud de l'île de Wight et au sud de Douvres. Des zones turbides apparaissent également le long des côtes françaises, vraisemblablement liées aux panaches de la Seine et de la Somme.

La présente étude vise à analyser à l'aide de codes numériques la capacité des courants de marée et de la houle à mettre en suspension les sédiments fins à moyens et à les advecter en Manche Orientale. Afin de faciliter cette analyse, l'impact d'agents a priori secondaires comme le vent et les gradients thermohalins est négligé. L'article s'organise successivement selon les étapes suivantes :

- le modèle hydro-sédimentaire et le modèle de propagation de houle sont brièvement décrits à la section 2 ,

- les résultats des modèles et leur confrontation avec les mesures in situ sont présentés section 3, ainsi qu'une description synoptique des influences respectives des courants de marée et d'une houle de tempête sur les mises en suspension,

- la section 4 synthétise les résultats.

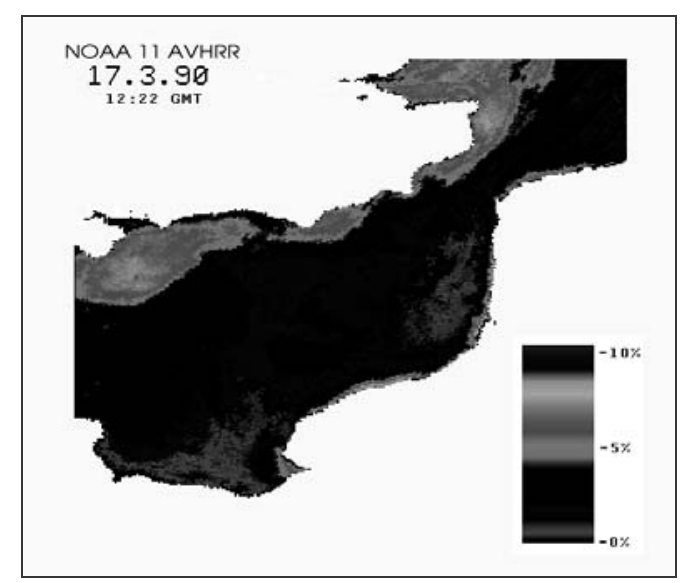

Figure 1: Image NOAA-AVHRR de la Manche Orientale présentant la réflectance de surface le 17.03.1990 (Kergomard et al., 1993)

\section{Présentation des modèles numériques}

\section{2-1-Modèle hydro-sédimentaire}

Le modèle hydrosédimentaire utilisé est basé sur le code tridimensionnel de circulation côtière COHERENS (Coupled Hydrodynamical Ecological Model for Regionals and Shelf Seas) [Luyten et al., 1999] qui résout les équations de Navier-Stokes sous l'hypothèse d'hydrostaticité et l'approximation de 
Boussinesq, et calcule l'évolution temporelle des champs 3D de vitesse moyenne, d'énergie cinétique turbulente et de viscosité. Ce code numérique, dédié à l'origine à des problématiques environnementales, a été adapté à la résolution de l'advection et de la dispersion de particules sédimentaires non-cohésives de différentes tailles (approche multiclasse).

Le code numérique est implanté sur deux zones gigognes (figure 2):

- la zone 1 avec une résolution de $2 \mathrm{~km}$ couvrant la quasi-totalité de la Manche et la partie sud de la mer du Nord,

- la zone 2 avec un maillage de $500 \mathrm{~m}$ de résolution localisé sur le détroit du Pas-de-Calais.

L'élévation de la surface libre est imposée aux frontières ouvertes de la zone 1 en recomposant le signal à l'aide des composantes harmoniques produites par un modèle bidimensionnel horizontal $(2 \mathrm{DH})$, réduit au mode barotrope de COHERENS, appliqué au domaine épicontinental des îles britanniques. Les conditions aux limites ouvertes de la zone 2 résultent de la surface libre et des courants prédits sur la zone 1 .

Les conditions aux limites ouvertes sont imposées toutes les $10 \mathrm{mn}$ en 15 points de chaque frontière. Un algorithme de type splin cubique permet l'interpolation temporelle et spatiale des variables forçantes.

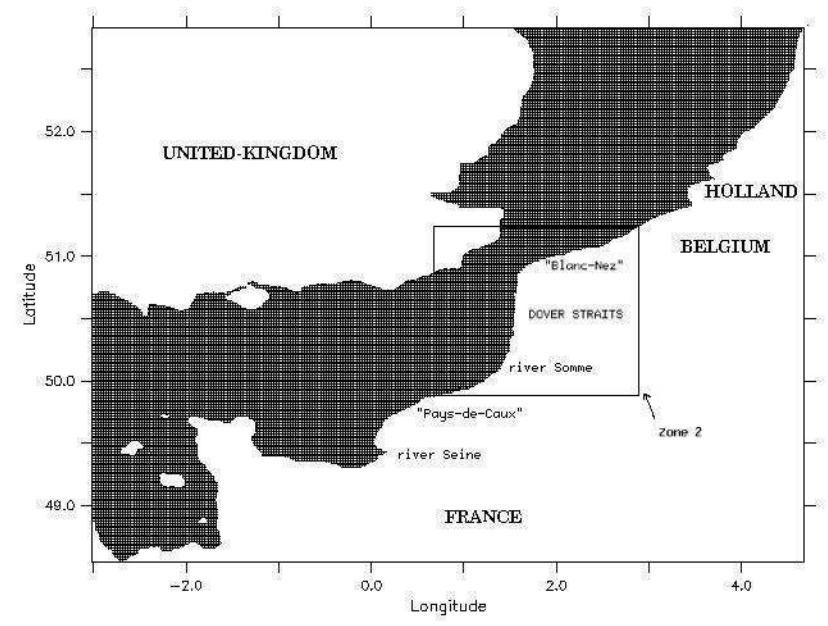

Figure 2: Emprise de la grille de calcul sur la zone 1. Le carré indique le domaine de calcul de la zone 2.

\section{2-2-Modèle de propagation de houle}

La modélisation des conditions locales de houle est réalisée à l'aide du code SWAN [Booij et Holthuijsen,1999] qui permet de prédire l'évolution des états de mer en zones côtières. Ceux-ci sont décrits, de manière statistique, en utilisant le spectre de houle. Le modèle résout ainsi l'évolution spatio-temporelle du spectre sur de larges échelles spatiales. Dans le cadre de la présente étude, le modèle de propagation spectrale de troisième génération SWAN a été implanté sur la zone 2. Ce code permet de disposer de conditions de houle en Manche Orientale et dans 
le détroit du Pas-de-Calais sur une grille compatible avec celle du modèle COHERENS.

\section{2-3- Modèle d'interaction houle-courant en couche limite benthique :}

L'action de la houle sur les mises en suspension est prise en compte au travers du phénomène d'interaction houle-courant en couche limite benthique. La modélisation est basée sur la théorie de Grant et Madsen (1979).

\section{Application}

\subsection{Cas des courants de marée seuls}

Mesures à points fixes

Les résultats du modèle sont comparés aux mesures effectuées en septembre 1997 par condition de mer belle, au niveau de deux sites littoraux, au large des plages de Hardelot (16,5 $\mathrm{m}$ de niveau moyen) et de Merlimont (13,5 m de niveau moyen) (figure 6) :

- par la Station d'Acquisition de Mesures Benthique Autonome (SAMBA) à 0,3, $0,6,0,9$ et $1,4 \mathrm{~m}$ au-dessus du fond,

- par un profileur acoustique de courant à effet Doppler BBADCP RDInstrument $(1200 \mathrm{kHz})$ dont les mesures réalisées à $5 \mathrm{~m}$ au-dessus du fond seront exploitées ici.

Plus de détails sont disponibles dans Chapalain et al. (1999, 2000).

Les figures 3 et 4 présentent respectivement les prédictions des composantes sudnord et ouest-est du courant de marée à 0,6 et $5 \mathrm{~m}$ au-dessus du fond à Merlimont, et les variations de l'énergie cinétique à 0,3 et $1,4 \mathrm{~m}$ au-dessus du fond à Hardelot. La figure 5 compare les résultats du modèle aux mesures de concentration à $5 \mathrm{~m}$ au-dessus du fond à Hardelot. On trouve un accord satisfaisant entre les mesures et les prédictions du modèle en termes d'amplitude et de phase des signaux.

\section{Champs synoptiques}

Les mises en suspension sont simulées au cours d'un cycle de marée moyenne (coefficient 70). L'étude de l'évolution spatio-temporelle des contraintes de cisaillement à proximité du fond révèle que seules les classes de diamètre médian $\mathrm{d}_{50}$ inférieur à $350 \mu \mathrm{m}$ sont transportées dans la colonne d'eau. Les mises en suspension simulées s'accordent avec les données compilées par Mc Cave (1973) et Eisma et Kalf (1993) dans les eaux centrales (moins de $8 \mathrm{mg} / \mathrm{l}$ ) et côtières (30 à $40 \mathrm{mg} / \mathrm{l}$ en moyenne). Les mises en suspension s'avèrent plus marquées en période de flot que de jusant tout particulièrement prés des côtes françaises. A titre d'exemple, au Nord-Est de Wissant, la concentration moyenne dans la colonne d'eau passe ainsi de $100 \mathrm{mg} / \mathrm{l}$ en jusant à $125 \mathrm{mg} / \mathrm{l}$ en période de flot. Ceci confirme un transit sédimentaire général orienté de la Manche à la mer du Nord avec des veines de transport plus marquées le long des côtes anglaises et françaises [Grochowski et al., 1993] (figures 6 et 7). Remarquons enfin l'apparition au cours du flot d'une zone de mise en suspension au Sud-Est du cap 
Gris Nez vraisemblablement due à l'accentuation des courants le long du flanc Est du cap.
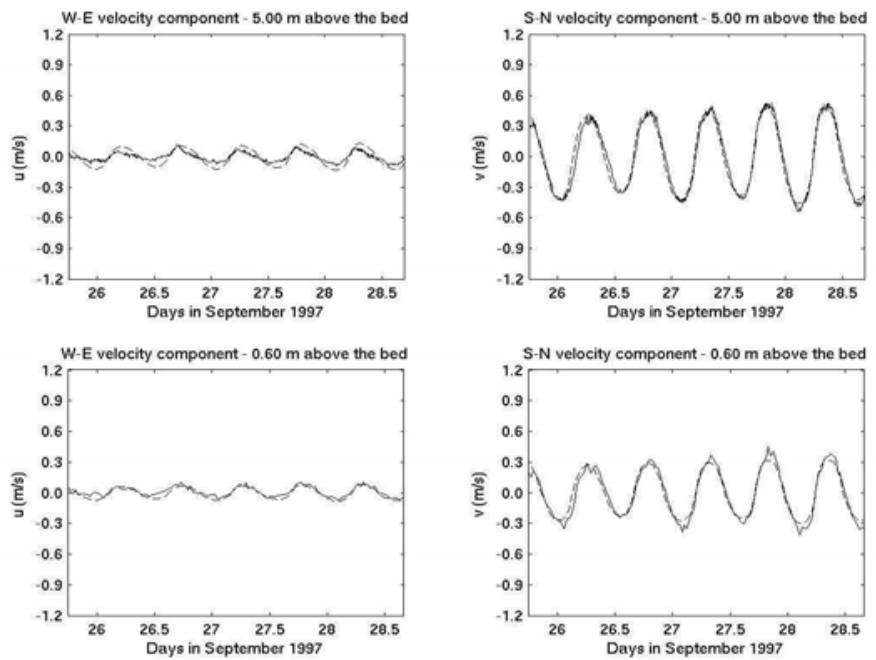

Figure 3: Séries temporelles de la composante sud-nord et ouest-est du courant à 0,6 et $5 \mathrm{~m}$ au-dessus du fond à Merlimont. La ligne continue correspond aux mesures, la ligne en pointillés aux prédictions du modèle.
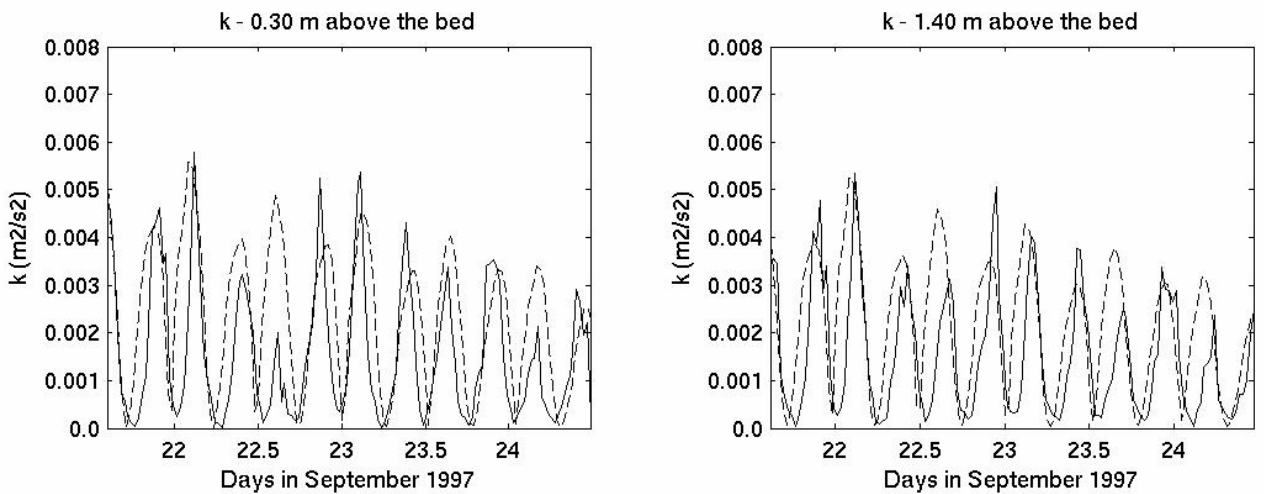

Figure 4: Séries temporelles de l'énergie cinétique turbulente à 0,3 et 1,4 $\mathrm{m}$ au-dessus du fond à Hardelot. La ligne continue correspond aux mesures, la ligne en pointillés aux prédictions du modèle.

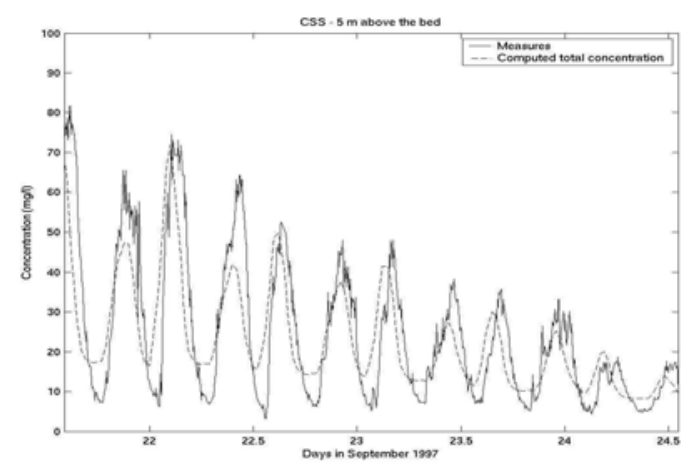

Figure 5 : Séries temporelles de la concentration totale en sédiments à $\mathbf{5} \mathbf{m}$ au-dessus du fond à Hardelot. La ligne continue correspond aux mesures, la ligne en pointillés aux prédictions du modèle. 


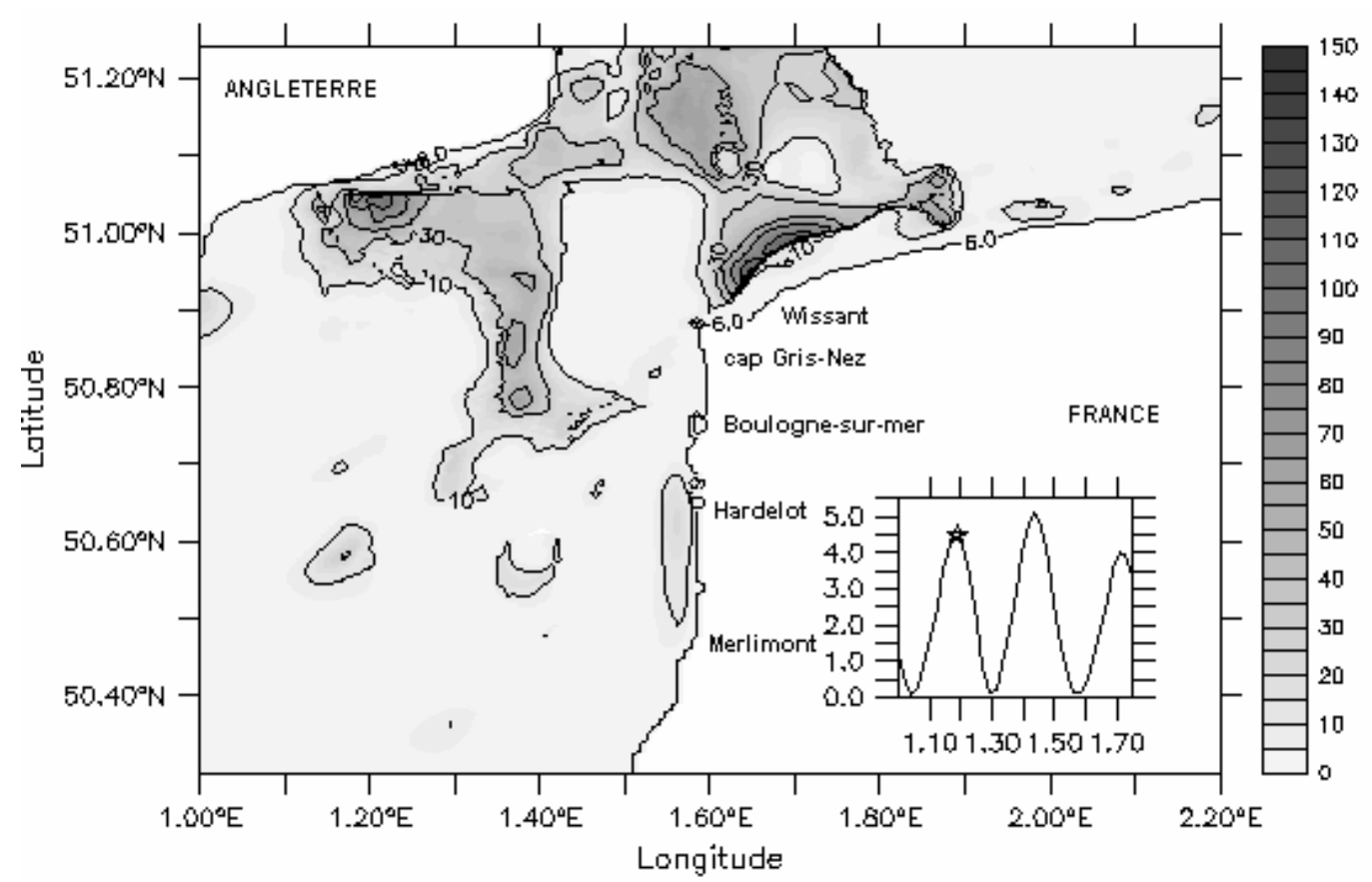

Figure 6: Champ synoptique de concentration moyenne en période de jusant (mg/l). La courbe en bas à gauche indique l'évolution du cisaillement au milieu du détroit (ordonnée : N/m², abscisse : jours).

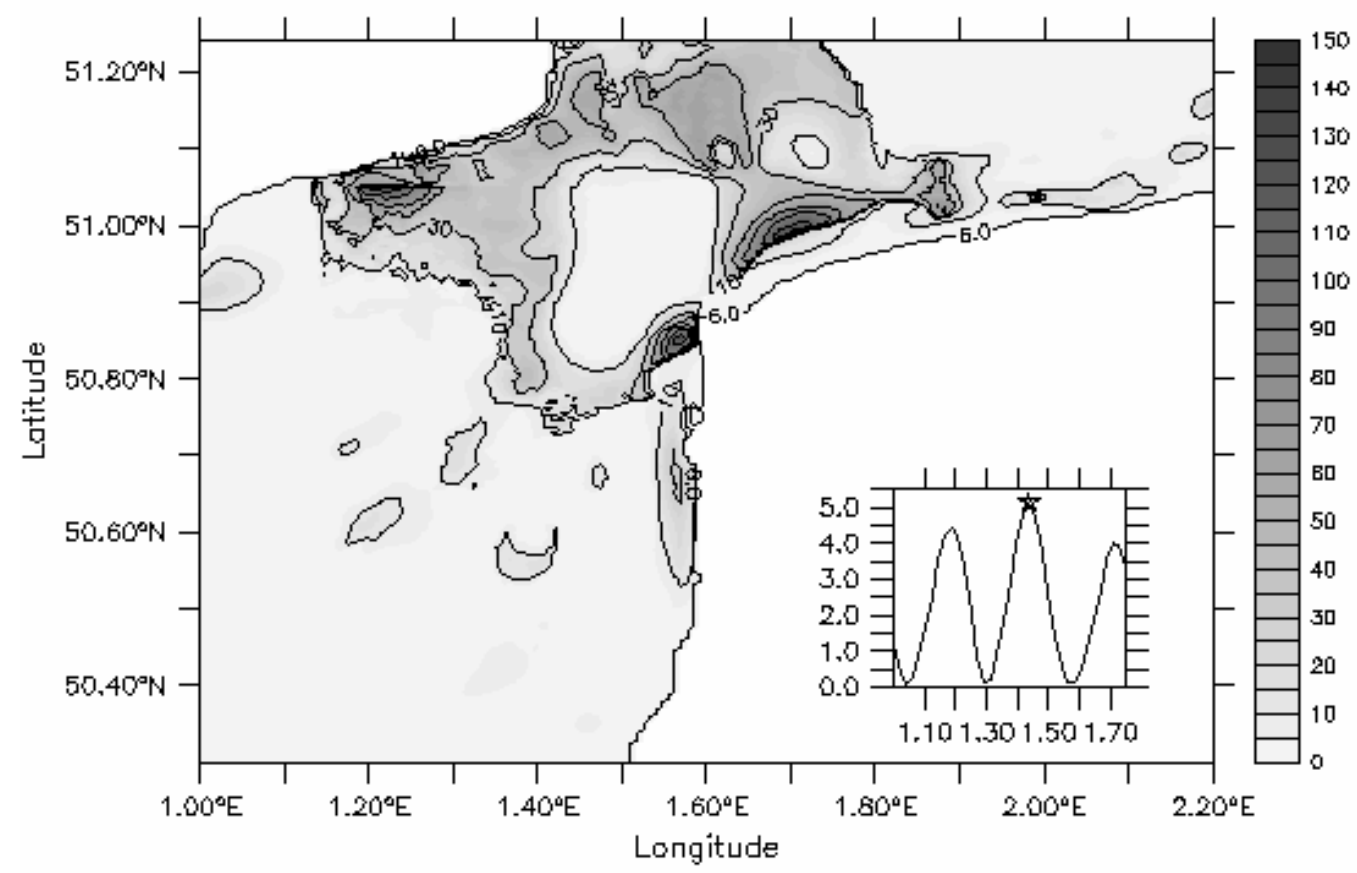

Figure 7: Champ synoptique de concentration moyenne en période de flot (mg/l). 


\subsection{Cas des courants de marée combinés à une houle de tempête}

Le détroit du Pas-de-Calais est une zone modérément exposée à la houle. Au cours de sa propagation en Manche Orientale ou dans la mer du Nord, l'énergie de la houle est progressivement dissipée par frottement sur le fond. Afin de tester l'influence de la houle sur les mises en suspension en présence de courants de marée, un cas de houle de tempête $\left(\mathrm{h}_{\mathrm{s}}=2,5 \mathrm{~m}, \mathrm{~T}_{\mathrm{p}}=6,5 \mathrm{~s}\right)$ a été simulé dans la zone 2. Ces conditions reprennent celles de Grochowski et al. (1993). L'analyse des directions de propagation entre 1997 et 2000 (cf. Atlas numérique de houle le long des côtes françaises ${ }^{1}$ ) montre que les houles provenant de la Manche Occidentale se propagent vers la mer du Nord selon l'axe médian au détroit $\left(54^{\circ}\right.$ mesuré par rapport à l'axe ouest-est dans le sens trigonométrique). La houle exacerbe globalement les mises en suspension dans la colonne d'eau (figure 8) gommant la typologie des concentrations générées par les courants de marée. Les principales zones de mises en suspension sont celles directement exposées à la houle; les maxima occupant la zone littorale de sables fins envasés facilement mobilisables située entre Merlimont et Hardelot. En période de flot, la concentration moyenne passe, sous l'effet d'une houle de tempête, de $11,5 \mathrm{mg} / 1$ à $60 \mathrm{mg} / \mathrm{l}$ au centre de cette zone.

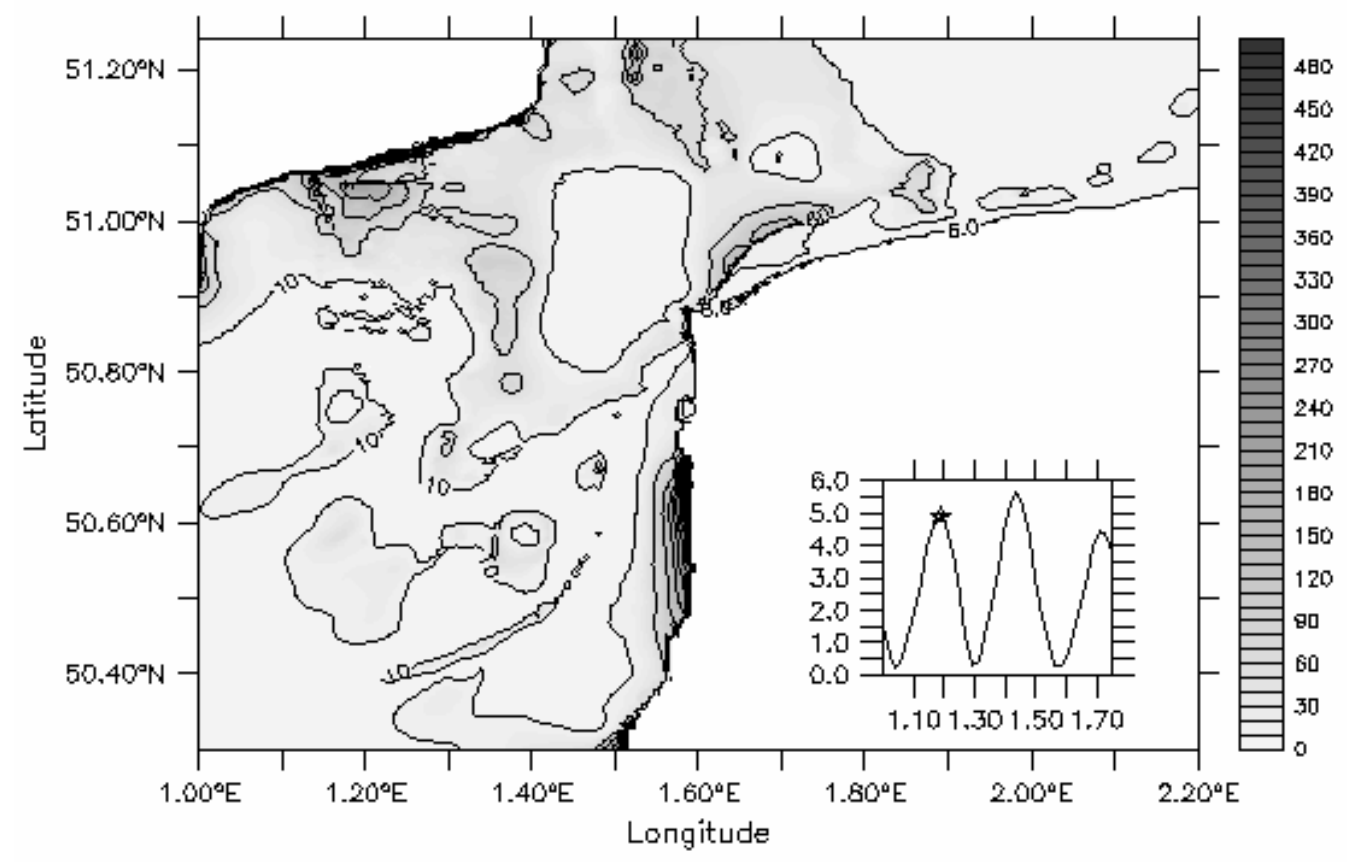

Figure 8: Champ synoptique de concentration moyenne en période de jusant en présence d'une houle d'Ouest (mg/l).

\section{Conclusion}

L'utilisation du modèle hydro-sédimentaire 3D COHERENS dans le détroit du Pas-de-Calais approche de manière satisfaisante les mesures réalisées en deux sites peu profonds. Le calcul des concentrations dans la colonne d'eau a permis de

\footnotetext{
${ }^{1}$ Projet commun Météo-France, CETMEF et EDF-LNHE.
} 
localiser les zones de mises en suspension sous l'effet d'une marée moyenne. Si la houle demeure modérée dans la zone d'étude, elle joue un rôle non négligeable sur les mises en suspension tout particulièrement à proximité du littoral Français situé au sud de Boulogne-sur-mer. L'allongement des durées de simulation permettrait de préciser l'impact de la houle sur les transits sédimentaires du détroit du Pas-de-Calais.

\section{Remerciements :}

Les auteurs tiennent à remercier Claude Kergomard ${ }^{2}$ pour la mise à disposition des données SPOT de réflectance de surface, ainsi que Michel Benoît ${ }^{3}$ pour l'extraction et la mise à disposition des données de l'atlas numérique de houle.

\section{Références :}

1 Booij N.R.C. and Holthuijsen L.H. (1999). A third generation wave model for coastal regions, Part I, Model description and validation. Journal of Geophysical Research, 104, C4, 7649-7666.

2 Chapalain G.,Thais L. and Smaoui H. (1999). Modeling of a tidal bottom boundary layer with suspended sediment. Hydrobiologia, 414, 1-12.

3 Chapalain G. and Thais L. (2000). Tide, turbulence and suspended sediment modelling in the eastern English Channel. Coastal Engineering, 41, 295-316.

4 Eisma D. and Kalf J. (1979). Distribution and particle size of suspended matter in the Southern Bight of the North Sea and the Eastern Channel. Netherlands Journal of Sea Research, 13, 2, 298-324.

5 Grant W.D. and Madsen O.S. (1979). Combined wave and current interaction with a rough bottom. Journal of Geophysical Research, 84, C4, 1797-1808.

${ }^{6}$ Grochowski N.T.L., Collins M.B., Boxall S.R., Salomon J.-C., Breton M. and Lafite R. (1993). Sediment transport pathways in the eastern English Channel. Oceanologica Acta, 16, 5-6.

${ }^{7}$ Kergomard C., De Luca D., Dilligeard E., Santer R. (1993). Télédétection et optique marine, Rapport LOADYSCOP.

8 Le Provost C. and Fornerino M. (1985). Tidal spectroscopy of the English channel with a numerical model. Journal of Physical Oceanography, 1009-1031.

9 Luyten P.J., Jones J. E., Proctor R., Tabor A., Tett P. and Wild-Aden K. (1999). Coherens: A coupled hydrodynamics-Ecological Model for Regionals and Shelf Seas - Part III Model Description.

${ }^{10}$ Mc Cave I. N. (1973). Mud in the North Sea. North Sea Science. Goldberg (Eds), M.I.T Press, 75-100.

\footnotetext{
${ }^{2}$ Laboratoire de géographie des milieux anthropisés, Ecole Nationale Supérieure

${ }^{3}$ EDF-LNHE
} 